\title{
Acute urticaria: an extremely rare adverse effect of levonorgestrel-releasing intrauterine system as a possible manifestation of progestogen hypersensitivity syndrome
}

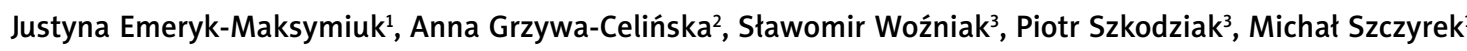 \\ ${ }^{1}$ Chair of Internal Medicine and Department of Internal Medicine in Nursing, Medical University of Lublin, Lublin, Poland \\ ${ }^{2}$ Chair and Department of Pneumology, Oncology and Allergology, Medical University of Lublin, Lublin, Poland \\ ${ }^{3} 3^{\text {rd }}$ Chair and Department of Gynecology, Medical University of Lublin, Lublin, Poland
}

Adv Dermatol Allergol 2018; XXXV (5): 530-531

DOI: https://doi.org/10.5114/ada.2018.75748

A 34-year-old Caucasian woman underwent insertion of a levonorgestrel-releasing intrauterine system (Mire$n a^{\circledR}$ ) for contraception in March 2016. The patient was in good general health and was menstruating regularly. The routine gynecological examination before the insertion of Mirena ${ }^{\circledR}$ was normal. Cytology and ultrasound examinations of the reproductive organs were also normal. The patient had a history of allergic rhinoconjunctivitis to birch, alder and hazel pollens. Before the insertion of Mirena ${ }^{\circledR}$ she was taking oral antihistamine and nasal glucocorticosteroid in typical doses for about 10 days to relieve the symptoms of allergic rhinoconjunctivitis as it was the pollen season of alder and hazel. On the implantation day, the patient did not drink alcohol and avoided strenuous exercises.

Mirena ${ }^{\circledR}$ was inserted according to the manufacturer's instructions without any complications. Two hours

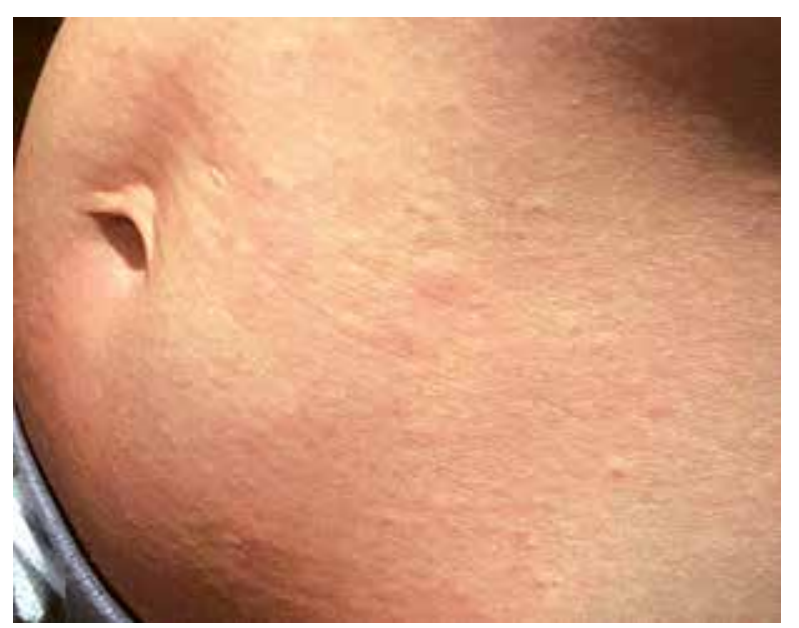

Figure 1. Urticaria after Mirena ${ }^{\circledR}$ after the procedure, the patient felt slightly dizzy and developed erythematous skin rash on her thighs, abdomen, chest and buttocks. Within the next $2 \mathrm{~h}$, typical wheals occurred on the abdomen (Figure 1) and she developed pruritus of the feet. Her general condition was good, vital signs were normal; there were no respiratory or gastrointestinal symptoms.

The patient's condition was diagnosed by an allergologist as "acute urticaria probably caused by an allergic reaction". She was advised to remove Mirena ${ }^{\circledR}$ as fast as possible and to take $20 \mathrm{mg}$ of oral methylprednisolone and $180 \mathrm{mg}$ of fexofenadine for 5 days. On the same day in the evening, Mirena ${ }^{\circledR}$ was removed. The gynecological examination did not reveal any skin rash, or mucosal congestion or edema of the urogenital region. The removed device showed no visible evidence of damage. The next day, the patient's skin condition improved; urticaria resolved completely two days after the removal of Mirena ${ }^{\circledR}$ and treatment with aforementioned medications. Urticaria did not recur.

The levonorgestrel-releasing intrauterine system is a long-term, highly effective, safe and reversible method of contraception [1, 2]. According to the manufacturer's information, the skin rash or/and urticaria are classified as rare $(\geq 1 / 10000$ to $<1 / 1000)$ adverse events observed during the use of Mirena ${ }^{\circledR}$ [3]. Cox et al. [4] in their study, which involved the population of 678 women with the device, report that its removal before the 5 -year period of use was required in 92 cases, yet only in one case its removal was associated with an allergic reaction (the type not specified).

The findings reported in the only available case report of urticaria after Mirena ${ }^{\circledR}$ implantation were comparable to our case. Chen et al. [5] have described a case of acute urticaria which developed about $2 \mathrm{~h}$ after the Mirena ${ }^{\circledR}$

Address for correspondence: Justyna Emeryk-Maksymiuk, MD, PhD, Chair of Internal Medicine and Department of Internal Medicine in Nursing, Medical University of Lublin, 8 Jaczewskiego St, 20-954 Lublin, Poland, phone: +48 8172447 02, e-mail: justynaemeryk@poczta.onet.pl Received: 6.03.2017, accepted: 9.07.2017. 
insertion and was accompanied by pruritus, slight dizziness and slight left lower quadrant pain. The patient was in good general condition, with normal vital signs. Mirena ${ }^{\circledR}$ was removed immediately. Three days after its removal and treatment with oral methylprednisolone and desloratadine, pruritus and abdominal pain disappeared and the skin condition improved significantly. Urticaria did not recur. Pereira et al. [6] have described another case of a skin adverse reaction to Mirena ${ }^{\circledR}$. One day after the insertion of Mirena ${ }^{\circledR}$, their patient developed an itchy eruption on her legs, spreading to the chest and arms. The diagnosis was "autoimmune progesterone dermatitis" and after initial alleviation of symptoms with local steroids and oral antihistaminics, the skin changes recurred and, finally, the coil had to be removed 45 days after implantation.

It is possible that all described cases are the manifestation of the same syndrome called "progestogen hypersensitivity". It is triggered by either endogenous progesterone (luteal phase of the menstrual cycle or pregnancy), either exogenous progestogens (any progesterone source not occurring naturally, including intrauterine devices). Its symptoms are heterogeneous and include cyclic dermatitis, urticaria, angioedema, asthma and anaphylaxis [7]. The pathophysiologic processes underlying progestogen hypersensitivity are type I or type IV hypersensitivity reaction mediated by lymphocytes Th2 [8]. In 50\% of the cases of progestogen hypersensitivity syndrome, it is accompanied by positive results of a skin prick and intradermal skin testing to progesterone [7]. Approaches to management of progestogen hypersensitivity vary greatly, depending on symptoms presented by patients and future plans for pregnancy. Symptomatic treatment includes topical or systemic corticosteroids and antihistaminics [9]. Suppression of endogenous progesterone by hormone antagonists [10] and agonists [11] or bilateral oophorectomy [12] are further therapeutic options. For patients with progestogen hypersensitivity who are planning pregnancy, there are oral and intramuscular protocols of desensitization to progestogens [7, 9].

Polyethylene glycol, which is an ingredient of lubricating gel used for transvaginal ultrasound, is another rare allergen that can cause anaphylactic reactions in gynecologic patients. Jakubovic et al. described a case of a woman who developed a progressive multisystem anaphylactic reaction after transvaginal ultrasound which was caused by polyethylene glycol [13].

Our case report of acute urticaria probably associated with Mirena ${ }^{\circledR}$ implantation is to raise awareness of side effects of this medication and progestogen hypersensitivity syndrome. Healthcare providers should be aware that even routine medical interventions might have potentially fatal complications related to hypersensitivity reactions.

\section{Acknowledgments}

The department to which the work should be contributed: Chair of Internal Medicine and Department of Internal Medicine in Nursing, Medical University of Lublin.

\section{Conflict of interest}

The authors declare no conflict of interest

\section{References}

1. Mansour D. The benefits and risks of using a levonorgestrelreleasing intrauterine system for contraception. Contraception 2012; 85: 224-34.

2. Hardeman J, Weiss BD. Intrauterine devices: an update. Am Fam Physician 2014; 89: 445-50.

3. Mirena - product information. Available at: http://www. bayerresources.com.au/resources/uploads/PI/file9399.pdf

4. Cox M, Tripp J, Blacksell S. Clinical performance of the levonogestrel intrauterine system in routine use by the UK Family Planning and Reproductive Health Research Network: 5-year report. J Fam Plann Reprod Health Care 2002; 28: 73-7.

5. Chen X, Wu X, Zhu H. Acute urticaria as a side effect of the Mirena ${ }^{\oplus}$ (levonorgestrel-releasing intrauterine system): a case report. BMC Res Notes 2014; 7: 209.

6. Pereira A, Coker A. Hypersensitivity to Mirena - a rare complication. J Obstet Gynaecol 2003; 23: 81.

7. Foer D, Buchkeit KM, Gargiulo AR, et al. Progesteron hypersensitivity in 24 cases: diagnosis, management and proposed renaming and classification. J Allergy Clin Immunol Pract 2016; 4: 723-9.

8. Cristaudo A, Bordignon V, Palamara F, et al. Progesterone sensitive interferon-gamma producing cells detected by ELISpot assay in autoimmune progesterone dermatitis. Clin Exp Dermatol 2007; 32: 439-41.

9. Nguyen T, Razzague A. Autoimmune progesterone dermatitis: update and insights. Autoimmun Rev 2016; 15: 191-7.

10. Shahar E, Bergman R, Pollack S. Autoimmune progesterone dermatitis: effective prophylactic treatment with danazol. Int J Dermatol 1997; 36: 708-11.

11. Fournier JB. Resolution of autoimmune progesterone dermatitis after treatment with oral contraceptives. JAAD Case Rep 2015; 25: 319-20.

12. Medeiros S, Rodrigues-Alves R, Costa M, et al. Autoimmune progesterone dermatitis: treatment with oophorectomy. Clin Exp Dermatol 2010; 35: 12-3.

13. Jakubovic BD, Saperia C, Sussman GL. Anaphylaxis following a transvaginal ultrasound. Allergy Asthma Clin Immunol 2016; 12: 3. 